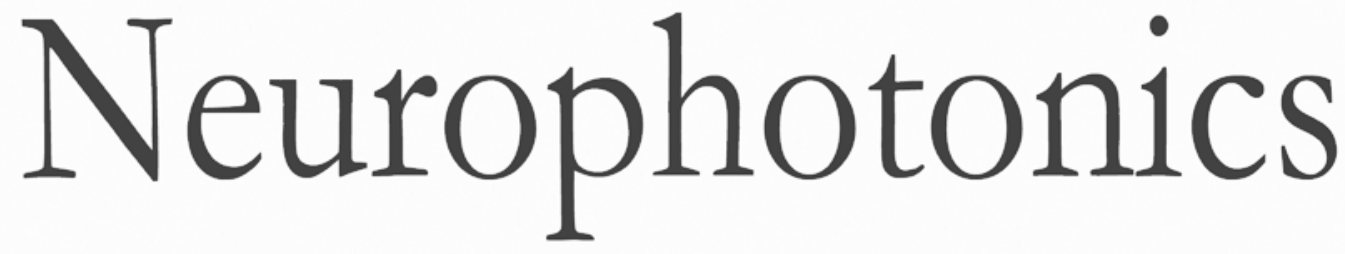

\title{
Infrared imaging: a potential powerful tool for neuroimaging and neurodiagnostics
}

Arezou Khoshakhlagh

Sarath D. Gunapala 


\title{
Infrared imaging: a potential powerful tool for neuroimaging and neurodiagnostics
}

\author{
Arezou Khoshakhlagh* and Sarath D. Gunapala \\ Jet Propulsion Laboratory, California Institute of Technology, Pasadena, California, United States
}

\begin{abstract}
Infrared (IR) imaging is used to detect the subtle changes in temperature needed to accurately detect and monitor disease. Technological advances have made IR a highly sensitive and reliable detection tool with strong potential in medical and neurophotonics applications. An overview of IR imaging specifically investigating quantum well IR detectors developed at Jet Propulsion Laboratory for a noninvasive, nonradiating imaging tool is provided, which could be applied for neuroscience and neurosurgery where it involves sensitive cellular temperature change. $\odot 2017$ Society of Photo-Optical Instrumentation Engineers (SPIE) [DOI: 10.1117/1.NPh.4.1.011014]
\end{abstract}

Keywords: quantum wells; infrared detectors; focal plane arrays; medical imaging.

Paper 16048SSR received Feb. 7, 2017; accepted for publication Mar. 14, 2017; published online Mar. 28, 2017.

\section{Introduction}

Infrared (IR) imaging and its application in medicine has been around since the early 1970s. Intraoperative IR brain imaging has been used to determine temperature-sensitive processes. Improvements in IR imaging and detection systems have allowed objective analysis of digital radiometric information for a variety of medical applications. IR imaging in medicine provides a noninvasive and nonradiating analysis tool for analyzing physiological functions related to the control of skin temperature. ${ }^{1}$ This rapidly developing technology is used to detect and locate thermal abnormalities characterized by an increase or decrease in temperature found at the skin surface. The technique involves the detection of IR radiation that can be directly correlated with the temperature distribution of a defined body region. This article describes real examples of biological and tissue imaging in the IR to establish its applicability and sensitivity framework for brain imaging. Highly uniform and sensitive IR imaging techniques developed at our laboratory at Jet Propulsion Laboratory (JPL) are described. Cases of cancer detection and a case of brain imaging for tumor delineation using this technology are discussed. Future directions are briefly described.

\section{Examples of Infrared Imaging Technologies for Tissue Imaging}

IR detector technology has been applied in a variety of medical imaging, for example, breast cancer risk assessment, coronary artery bypass surgery, analysis of burn trauma, diabetes, and brain tumor delineation.

IR cameras have helped breast cancer risk assessment tremendously. In a prospective study of 58,000 women being screened for breast cancer, there were 784 patients that had an abnormal asymmetric IR image of their breasts, 298 (38\%) of these 784 patients were diagnosed with breast cancer within four years. ${ }^{2}$

*Address all correspondence to: Arezou Khoshakhlagh, E-mail: Arezou. khoshakhlagh@jpl.nasa.gov
IR technology has shown great potential for differentiating a second-degree burn from a third-degree burn. ${ }^{3}$ Many burn patients for whom the physician cannot initially determine whether the burn is a second- or third-degree burn have to wait 5 to 7 days before a decision. If done by IR imaging, the average hospital time for burn patients could be significantly reduced. IR imaging on 10 patients with burns that could not be staged by visual analysis, that is, the burns could be either second or third degree, were performed. They found that in third-degree burns, the area of the burn had reduced temperatures compared to the surrounding normal unburned skin but that an obvious seconddegree burn had an increased temperature probably due to inflammation.

Another application of IR imaging technology is in the prevention and management of diabetic foot disease. IR detectors analysis demonstrates that there is a relationship between increased temperature and foot complications in diabetes, increased temperature may be present up to a week before a foot ulcer occurs. ${ }^{4}$ Temperature of corresponding area of the right and left foot does not usually differ more than $1{ }^{\circ} \mathrm{C}$ in diabetic foot. A temperature difference greater than $2.2^{\circ} \mathrm{C}$ is considered abnormal. Detecting increased temperature between the two feet and providing adequate therapy can reduce the incidence of a foot ulcer significantly. The results of this study have made several research teams, sometimes associated to a private company, propose or test at home devices to monitor foot temperature to detect and prevent a foot ulcer. ${ }^{5}$

Depending on the wavelength and sensitivity of the experiment, different IR detector technologies can be used for the medical applications mentioned above. In this work, we will focus on quantum well IR detectors (QWIP) that have high uniformity and sensitivity at lower temperatures.

\section{Quantum Well Infrared Photodetector as a Powerful IR Imaging Tool}

A quantum well designed to detect IR light is called a QWIP. In recent years, many research groups in the world have demonstrated large format QWIP focal plane arrays (FPAs) for various

$2329-423 X / 2017 / \$ 25.00$ (c) 2017 SPIE 
thermal imaging applications. QWIPs are well-known to provide high uniformity, large, reproducible, sensitivity, and lowcost FPAs with few bad pixels. Moreover bandgap engineering allows it to reach any desired wavelength between 3 and $70 \mu \mathrm{m}$. The flexibility of wide range of cutoff wavelengths gives the ability of detection performance in environments with different temperatures, which is helpful for detection of a variety of medical diagnosis with different backgrounds/temperatures. QWIPs, as opposed to conventional low bandgap IR detectors, are limited by thermionic dark current and not tunneling currents down to $30 \mathrm{~K}$ or less. As a result, the performance of QWIPs can be substantially improved (orders of magnitude) by cooling from 70 to $30 \mathrm{~K}$. Cooling does not induce any nonuniformity or $l / f$ noise in QWIP FPAs. Compared to other IR technology, QWIPs offer high uniformity, high stability, and large format $[1 \mathrm{~K} \times$ $1 \mathrm{~K}$ long wavelength IR (LWIR) FPA with noise equivalent differential temperature of $13 \mathrm{mK}$ has been reported]. ${ }^{6}$

In the following paragraph, the detailed structure, physics, and manufacturing of the QWIP cameras developed at JPL and used for medical applications are discussed.

It is customary to make IR detectors in the long wavelength range ( 8 to $20 \mu \mathrm{m}$ ) by utilizing the interband transition, which promotes an electron across the bandgap $(E g)$ from the valence band to the conduction. These photoelectrons can be collected efficiently, thereby producing a photocurrent in the external circuit. Since the incoming photon has to promote an electron from the valence band to the conduction band, the energy of the photon $(h v)$ must be higher than the $E g$ of the photosensitive material. Therefore, the spectral response of the detectors can be controlled by manipulating $E g$ of the photosensitive material. Detection of LWIR radiation up to $20 \mu$ m requires small bandgaps down to $62 \mathrm{meV}$. Examples of such materials meeting these requirements are $\mathrm{Hg}_{1-x} \mathrm{Cd}_{x} \mathrm{Te}$ and $\mathrm{Pb}_{1-x} \mathrm{Sn}_{x} \mathrm{Te}$ in which the energy gap can be controlled by varying $x$. It is well-known that these low bandgap materials are more difficult to grow and process than large bandgap semiconductors, such as GaAs. These difficulties motivate the exploration of utilizing the intersubband transitions in multiquantum well (MQW) structures made of large bandgap semiconductors (Fig. 1).

The detection mechanism of the QWIP involves photoexcitation of electrons between ground and first excited state subbands of MQWs that are artificially fabricated by placing thin layers of two different, high-bandgap semiconductor materials alternately. ${ }^{7,8}$ The bandgap discontinuity of the two materials creates quantized subbands in the potential wells associated with conduction bands or valence bands. The structure parameters are designed so that the photo excited carriers can escape from the potential wells and be collected as photocurrent.

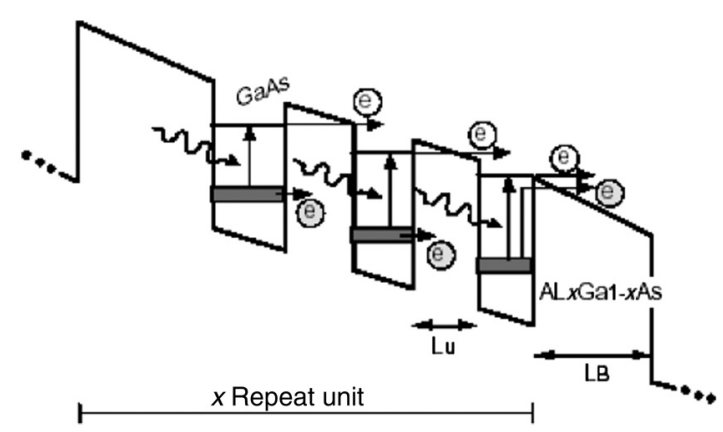

Fig. 1 Schematic of GaAs/AIGaAs QWIP IR detector.
In addition to larger intersubband oscillator strength, these detectors offer greater flexibility than the usual extrinsically doped semiconductor IR detectors because the wavelength of the peak response and cutoff can be continuously tailored by varying layer thickness (well width) and barrier composition (barrier height).

The lattice matched $\mathrm{GaAs} / \mathrm{Al}_{x} \mathrm{Ga}_{l-x}$ As material system is commonly used to create such a QWIP structure. Highly uniform and pure crystal layers of such semiconductors can be grown on large substrate wafers, ${ }^{9}$ with control of each layer thickness down to a fraction of a molecular layer, using crystalgrowth methods, such as molecular beam epitaxy. Thus, by changing the quantum well width and the barrier height (Al molar ratio of $\mathrm{Al}_{x} \mathrm{Ga}_{l-x} \mathrm{As}$ alloy), this intersubband transition energy can be varied over a wide enough range to enable light detection at any narrow wavelength range between 6 and $20 \mu \mathrm{m}$.

QWIP cameras can be made for different wavelengths and formats depending on the application they are being used for. There are many applications that require medium wavelength IR (MWIR) and LWIR dual-band FPAs. For example, a dualband FPA camera would provide the absolute temperature of a target with unknown emissivity, which is extremely important for the process of identifying temperature difference between missile targets, warheads, and decoys. Dual-band IR FPAs can also play many important roles in Earth and planetary remote sensing, astronomy, etc. Furthermore, monolithically integrated pixel collocated simultaneously readable dual-band FPAs eliminate the beam splitters, filters, moving filter wheels, and rigorous optical alignment requirements imposed on dualband systems based on two separate single-band FPAs or a broadband FPA systems with filters. Dual-band FPAs will also reduce the mass, volume, and power requirements of dual-band systems. Dual-band FPA developed at JPL is based on two different types (i.e., MWIR and LWIR) of QWIP devices separated [Fig. 2(a)] by a $0.5-\mu$ m-thick heavily doped n-type GaAs layer. The carriers emitted from each MWQ region are collected separately using three contacts. The middle contact layer is used as the detector common [detector Fig. 2(b)].

\section{Brain Tumor Delineation and Other Cancer Detection Using Intraoperative and Noninvasive Infrared Imaging Using Quantum Well Infrared Photodetector Technology}

LWIR QWIP cameras developed at the JPL, in collaboration with Raytheon Systems, demonstrate the potential of GaAs/ A1GaAs QWIP technology for highly sensitive, low-power, low-cost, and highly uniform large format FPA imaging systems. The hand-held LWIR camera features a JPL-developed $256 \times 256$ pixel, 8 to $9 \mu \mathrm{m}$ QWIP FPA mounted into a 450 $\mathrm{mW}$ Stirling closed-cycle cooler assembly, and installed into an Amber Radiance-1 camera body, ${ }^{10}$ see Fig. 3(a). The camera has 12-bit data resolution, weight less than $10 \mathrm{lb}$, consumes less than $50 \mathrm{~W}$, and features push-button control of all imaging parameters. A 32-bit floating-point digital signal processor combined with multitasking software executes image-processing and analysis functions within the camera body. The other element of the camera is a $100-\mathrm{mm}$ focal length germanium lens, with a 5.5-deg field of view. It is designed for transparence in the 8 - to $12-\mu \mathrm{m}$ wavelength range, to be compatible with the QWIPs $8.5 \mu \mathrm{m}$ operation. 
(a)



(b)

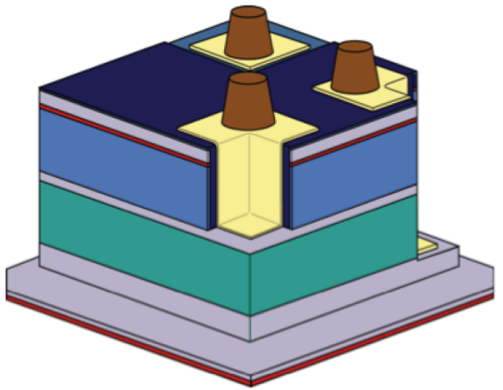

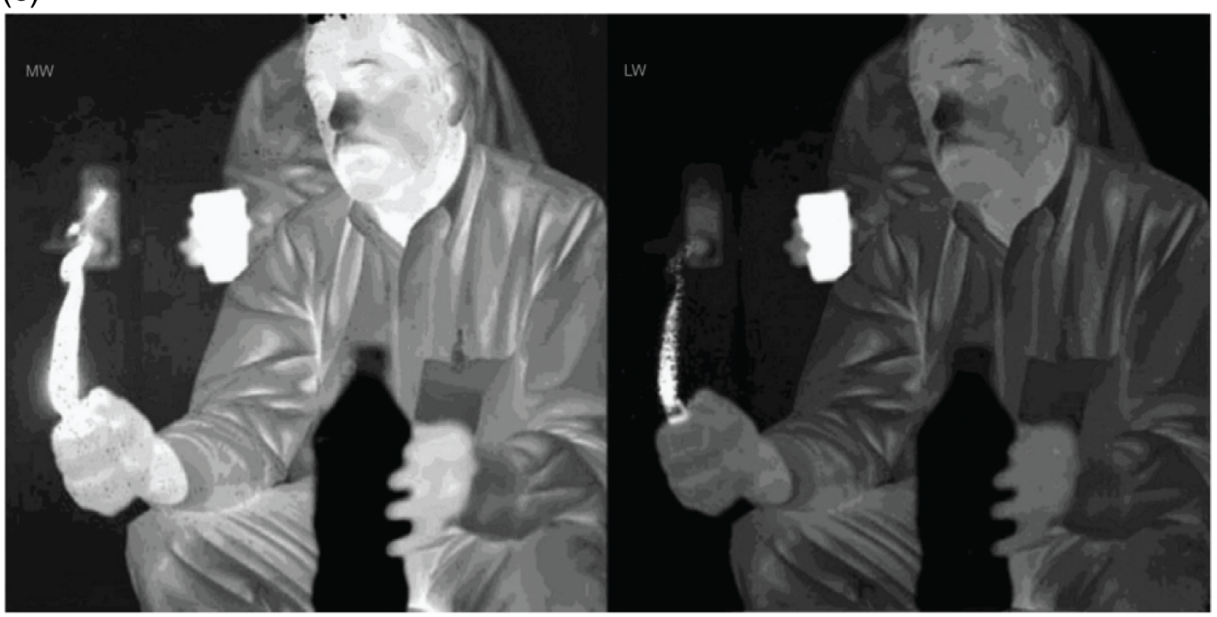

Fig. 2 (a) Two-dimensional view of dual-band QWIP device structure, (b) three-dimensional view of dual-band QWIP device structure showing via connects for independent access of MWIR and LWIR devices, and (c) IR image take by the dual-band QWIP at both MWIR and LWIR bands.

(a)

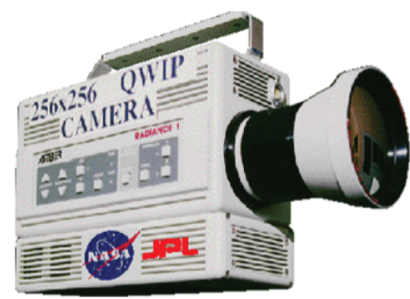

(b)
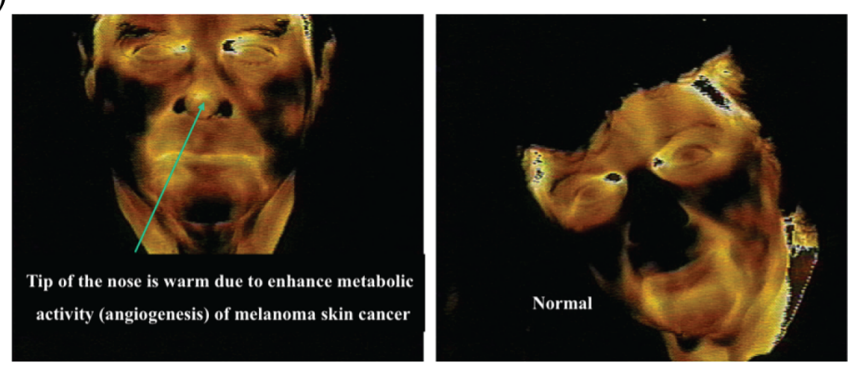

Fig. 3 (a) $256 \times 256$ hand-held long wavelength QWIP camera and (b) imagery of carcinoma (melanoma skin cancer) using JPL QWIP camera.

Such a portable LWIR camera can be used not only in classical night-vision applications but for medical imaging, such as thermal mapping of human skin and brain surgery.

IR cameras are used for resolving temperature differences in a system, and this can be an excellent approach for identifying diseases that display temperature gradients in humans through a variation in blood flow. Cancer cells have a different metabolism rate from healthy cells and in melanoma, ${ }^{11}$ for example, can show up as a temperature variation on the surface of the skin, which can be differentiated using IR imaging [Fig. 3(b)]. JPL's QWIP camera helped Dr. Norman E. Levan to diagnose a leprosy (a chronic infectious disease that primarily affects the peripheral nerves and skin) patient by thermally imaging the affected area of the skin (Fig. 4).

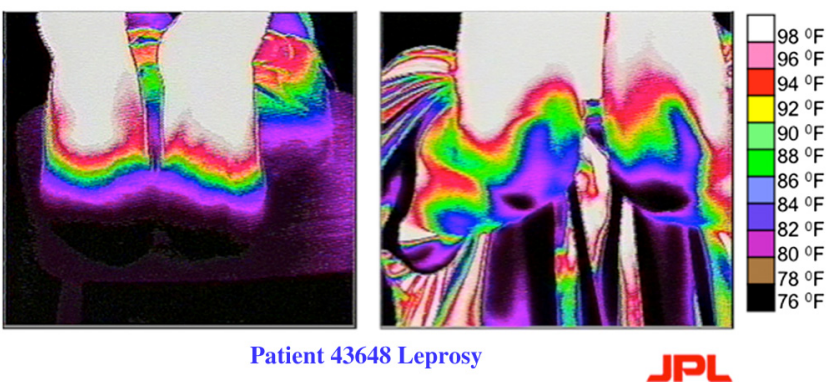

Fig. 4 The temperature variation of the toes and elbows of a leprosy patient. 


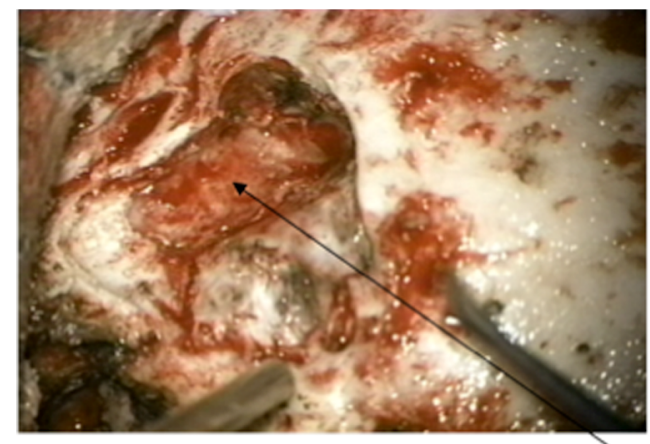

(a) Visible camera

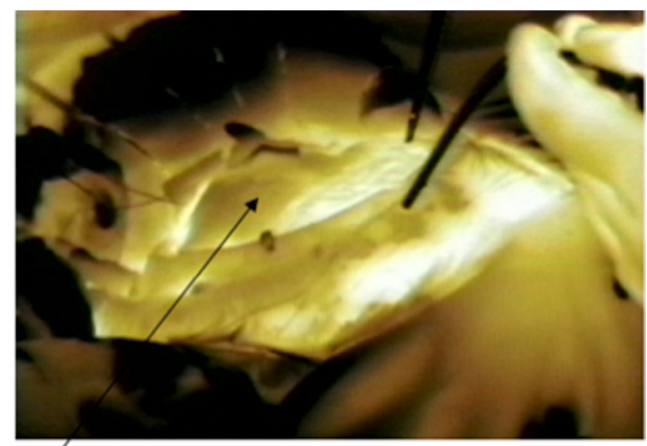

(b) QWIP IR camera

Fig. 5 Brain tumor image by (a) visible camera and by (b) QWIP IR camera.

The QWIP camera was used to monitor a patient with Schamberg's disease, which is a chronic discoloration of the skin that is caused by leaky blood vessels near the surface of the skin.

The QWIP also helped the University of Southern California brain surgeon Dr. Michael Levy to see the boundary of cancer and healthy tissue (Fig. 5). The IR camera assists the surgeon to remove blood supply vessels to tumor cells, which are the result of cancer promoted angiogenesis. The quality of the vascular connections can be monitored and checked during the surgery by the quantity of heat being brought to the newly attached blood vessels. Those boundaries are hard to differentiate in visible regime. The credibility and acceptance of thermal imaging in medicine are subject to critical use of the technology and proper understanding of thermal physiology.

JPL QWIP radiance camera has also been used by a group of researchers from the State University of New York in Buffalo and Walter Reed Army Institute of Research in Washington DC in the dynamic area telethermometry (DAT). ${ }^{12,13}$ DAT has been used to study the physiology and pathophysiology of cutaneous perfusion, which has many clinical applications. DAT involves accumulation of hundreds of consecutive IR images and fast Fourier transform (FFT) analysis of the biomodulation of skin temperature and of the microhomogeneity of skin temperature [spatial homogeneity of skin temperature (HST), which measures the perfusion of the skin's capillaries]. The FFT analysis yields the thermoregulatory frequencies and amplitudes of temperature and HST modulation. Their preliminary study demonstrates significant potential advantages of DAT over static thermal imaging as a diagnostic tool for breast cancer. To obtain reliable DAT data, one needs an IR camera in the $>8 \mu \mathrm{m}$ wavelength range with a repetition rate of $30 \mathrm{~Hz}$ [allowing accumulation of a maximal number of images during the observation period (to maximize the resolution of the FFT), frame-toframe instrumental stability (to avoid artifact stemming from instrument modulation), and sensitivity of less than $50 \mathrm{mK}$. 3 to $5 \mu \mathrm{m}$ cameras may not be suitable for quantitative clinical applications because of the reflectivity of the human skin in this wavelength range] to avoid artifacts of reflections of modulated emitters in the environment. ${ }^{14}$ According to these researchers, the longer wavelength operation, higher spatial resolution, higher sensitivity, and greater stability of the QWIP radiance made it the best choice of all IR cameras.

\section{Conclusion}

IR imaging was introduced into medicine in early 1970s. Although, the early instrumentation was not sensitive enough to detect the subtle changes in temperature needed to accurately detect and monitor disease. In recent years, the military has greatly improved the sensitivity of IR instruments. The further development of FPA IR sensors has allowed high-quality imaging as well as the export of digital temperature measurements amiable to computer-assisted image analysis and algorithm development. The medical community has also acquired stateof-the-art FPA instrumentation and is now beginning to investigate the applications of this instrumentation and computer-assisted image analysis to today's most difficult medical problems. These applications include breast cancer (risk assessment, detection, prognosis, and therapeutic monitoring), burn trauma (staging), diabetes, vascular problems, and neurological problems. Also, quantum well infrared detectors have shown as a promising high resolution, high sensitivity infrared imaging tool to be used for medical diagnostics.

\section{Disclosures}

No conflicts of interest, financial or otherwise, are declared by the authors.

\section{References}

1. S. E. Godoy et al., "Dynamic infrared imaging for skin cancer screening," Infrared Phys. Technol. 70, 147-152 (2015).

2. M. Gautherie and C. M. Gros, "Breast thermography and cancer risk prediction," Cancer 45(1), 51-56 (1980).

3. A. G. Hargroder et al., "Infrared imaging of burn wounds to determine burn depth," Proc. SPIE 3698, 103 (1999).

4. D. G. Armstrong et al., "Skin temperature monitoring reduces the risk for diabetic foot ulceration in high-risk patients," J. Med. 121(12), 1042-1046 (2008).

5. K. Roback, "An overview of temperature monitoring devices for early detection of diabetic foot disorders," Expert Rev. Med. Devices 7(5), 711-718 (2010).

6. S. D. Gunapala et al., "MWIR and LWIR megapixel QWIP focal plane arrays," Proc. SPIE 5563, 141 (2004).

7. S. D. Gunapala and K. M. S. V. Bandara, "Recent developments in quantum-well infrared photodetectors," Phys. Thin Films 21, 113-237 (1995).

8. B. F. Levine, "Quantum-well infrared photodetectors," J. Appl. Phys. 74(8), R1 (1993). 
9. S. V. Bandara et al., "10 $16 \mu \mathrm{m}$ broadband quantum well infrared photodetector," Appl. Phys. Lett. 72, 2427 (1988).

10. S. D. Gunapala et al., " $15-\mu \mathrm{m} 128 \times 128 \mathrm{GaAs} / \mathrm{AlxGa1-xAs}$ quantum well infrared photodetector focal plane array camera," IEEE Trans. Electron Devices 44(1), 45-50 (1997).

11. N. D. Amoêdo et al., "How does the metabolism of tumor cells differ from that of normal cells," Biosci. Rep. 33, 6 (2013).

12. M. Anbar, "Hyperthermia of the cancerous breast: analysis of mechanism," Cancer Lett. 84(1), 23-29 (1994).

13. M. Anbar, K. H. Eckhert, and L. Milescu "Preliminairary study of women's breasts with dynamic area telethermometry (DAT)," in Int. Congress of Thermology Conf., Ft. Lauderdale, Florida (1998).

14. A. Michel et al., "In vivo measurement of mid-infrared light scattering from human skin," Biomed. Opt. Express 4(4), 520-530 (2013).
Arezou Khoshakhlagh received her PhD in optical sciences and engineering from the University of New Mexico in 2010 where she worked on design, growth, and characterization of type-II strained layer superlattice infrared (IR) detectors. She joined the Infrared Focal Planes and Photonics Technology Group, Jet Propulsion Laboratory (JPL) in April 2010 and has been leading the material growth and material characterization of midwave IR, long-wave IR, and two color superlattice arrays.

Sarath D. Gunapala received his $\mathrm{PhD}$ in physics from the University of Pittsburgh in 1986. Since then he studied IR properties of III-V compound semiconductor heterostructures and the development of quantum well IR photodetectors for IR imaging at AT\&T Bell Laboratories. He joined NASA's JPL at California Institute of Technology in 1992. $\mathrm{He}$ is the director for Center for Infrared Photodetectors at JPL as well as a fellow of IEEE, SPIE, and OSA. 